\title{
Quit your YAPing: a new target for cancer therapy
}

\author{
Ben Z. Stanger ${ }^{1}$ \\ Gastroenterology Division, Department of Medicine, Department of Cell and Developmental Biology, Abramson Family Cancer \\ Research Institute, Perelman School of Medicine, University of Pennsylvania, Philadelphia, Pennsylvania 19104, USA
}

The Hippo pathway is an evolutionarily conserved signaling module that plays multiple roles in embryonic development. Components of the pathway, which includes a kinase cascade and a downstream complex composed of YAP and TEAD transcription factors, are dysregulated in a significant fraction of human cancers. In this issue of Genes \& Development, Liu-Chittenden and colleagues (pp. 1300-1305) use genetic and pharmacological means to disrupt the active YAP-TEAD complex. As this intervention impedes tumorigenesis in the liver with no apparent effect on normal liver homeostasis, the work paves the way for the development of new strategies to target this pervasive oncogenic pathway.

For much of the 20th century, treating cancer meant taking a broad swipe at tumors. By using "conventional chemotherapy" — an arsenal that includes radiation, alkylating agents, and anti-metabolites-oncologists were able to achieve numerous remissions and even some cures. But these clinical responses came at a significant cost, since conventional modalities act promiscuously to kill rapidly dividing cells-lack of specificity that results in damage to tissues with a high rate of turnover, particularly the intestines, skin, and blood. Hence, the side effects from chemotherapy and radiation-toxicities that limit the tolerated dose and cause complications (infection, cytopenias, and organ failure) that can be as lethal as the cancer itself-have limited the effectiveness of these approaches.

Over time, it became clear that some tumors exhibit intrinsic sensitivities to certain agents, allowing them to be attacked with greater precision than the "carpetbombing" approach of traditional therapies. For example, acute promyelocytic leukemia (APML), in which the use of all-trans retinoic acid induced a similarly high rate of remission by driving tumor cells to differentiate. In both of these tumor types, durable clinical responses with conventional chemotherapy alone had been quite rare. Such opportune observations in the 1970s and 1980s fueled an intensive effort to achieve a similar degree of

[Keywords: Hippo signaling; YAP; chemical biology; oncogene]

${ }^{1}$ Correspondence

E-mail bstanger@exchange.upenn.edu

Article is online at http://www.genesdev.org/cgi/doi/10.1101/gad.196501.112. specificity for other cancers. The goal was to develop drugs that would exploit unique biological properties of a tumor that made it selectively sensitive to modulation of a particular pathway or molecule. Ideally, the targets of these new therapies would have minimal roles in the homeostasis of normal tissues but would be essential for the survival and/or growth of cancer cells.

One of the first proof-of-principle experiments came from mouse models of breast cancer, in which treatment of tumor-bearing animals with a "designer" monoclonal antibody directed against the Her2/neu oncogene, a prototype for trastuzumab, was able to inhibit the growth of neu-transformed cancer cells (Drebin et al. 1986). Subsequently, a series of small molecule inhibitors and antibody antagonists directed against specific molecules has been generated. The molecular targets of this class of agents-which includes imatinib, erlotinib, bevacizamab, and rituximab-are largely known, and their dramatic efficacy in selected patient populations has brought us into the age of "targeted therapy" (http://www.cancer.gov/ cancertopics/factsheet/Therapy/targeted). Despite these impressive advances, however, the current array of targeted therapies has had a somewhat limited spectrum of efficacy, working only in those diseases where the target plays a critical nonredundant role. Hence, the next step in the evolution of targeted therapies would be the identification of pathways or molecules that are required for tumor growth in a wide variety of cancers, but whose role in normal tissues is minimal-a kind of "magic bullet" for anti-tumor therapy.

\section{The mammalian Hippo-YAP pathway}

Whether such global yet selective targets exist remains an open question, but an excellent candidate for such a magic bullet is the Hippo pathway, a newly delineated signaling module that drives cell proliferation and survival in a large fraction of tumors and yet seems to play a minimal role in adult tissue homeostasis. Components of the pathway were initially revealed through genetic screens in Drosophila for mutations that caused clonal overgrowth (Justice et al. 1995; Xu et al. 1995), and a series of biochemical and genetic studies over the ensuing decade revealed a pathway whose components are highly conserved between flies and mammals (for review, see Pan 
2010; Zhao et al. 2010a). The pathway is named after the Ste20-like protein kinase Hippo (Hpo), a central component of a regulatory kinase cascade. In mammals, the pathway is stimulated by the FERM domain-containing tumor suppressor NF2/Merlin, resulting in the sequential activation of two pairs of kinases: Mst1/2 (the mammalian orthologs of Hippo) and Lats1/2 (the mammalian orthologs of the Drosophila Warts kinase). The activity of these core kinases is further modulated by a number of other conserved proteins, including RASSF (Ras association domain family), Mob1, and Sav1. Ultimately, this regulated kinase cascade controls the transcriptional activity of a conserved nuclear complex composed of members of the TEAD family of DNA-binding proteins and the YAP transcriptional activator (Fig. 1). In contrast, the elements that lie upstream of NF2/Merlin-connecting extracellular inputs to the Hippo kinase cascade-do not exhibit strong functional conservation and have not been well characterized in mammals.

The Hippo pathway has many important roles in metazoan development, where it regulates not only growth, but also cell fate decisions and cell polarity (Halder and Johnson 2011), and the biochemical activities of pathway components seem also to be well conserved. Lats $1 / 2$ modulate YAP activity by phosphorylating it and directly regulating its cellular localization and stability. In the phosphorylated state (pathway ON), YAP is ubiquitinated and degraded (Zhao et al. 2010b) or is bound by 14-3-3 proteins, which sequester it in the cytoplasm (Zhao et al. 2007; Lei et al. 2008). In its unphosphorylated state (pathway OFF), YAP is free to migrate into the nucleus, where it binds to one of four TEAD transcription factors (TEAD1-4) (Sawada et al. 2008). TEAD proteins have limited transcriptional activity on their own, but in the presence of YAP, the complex becomes transcriptionally active, inducing transcription of several genes that are important in cell proliferation and survival. TAZ, a YAP homolog, is regulated through similar mechanisms and exhibits functional redundancy with YAP.

Based on the initial strategy used to identify pathway components (tumor formation in flies), one might have predicted that pathway orthologs play a role in mammalian tumorigenesis-and indeed, examples of such a role are abundant (Fig. 1). The best described of these is NF2/ Merlin, a bona fide tumor suppressor gene whose loss is responsible for neurofibromatosis, an autosomal dominant condition characterized by a wide spectrum of intracranial and spinal cord tumors (Asthagiri et al. 2009). Aside from NF2/Merlin, mutations and deletions in upstream Hippo pathway components are rare. Thus, tumors use other mechanisms for down-regulation of upstream regulatory components - the Mst1/2 and Lats1/2 kinases and their regulators, RASSF, WW45, and Mob1-to activate the YAP/TAZ-TEAD transcription complex, including gene hypermethylation and microRNA silencing. Although loss of these genes does not occur with high frequency in human tumors, murine gene targeting studies have confirmed that Mst1/2, Lats1/2, and WW45 act as tumor suppressor genes in vivo (for review, see Pan 2010; Zhao et al. 2010a). YAP itself functions as an oncogene in

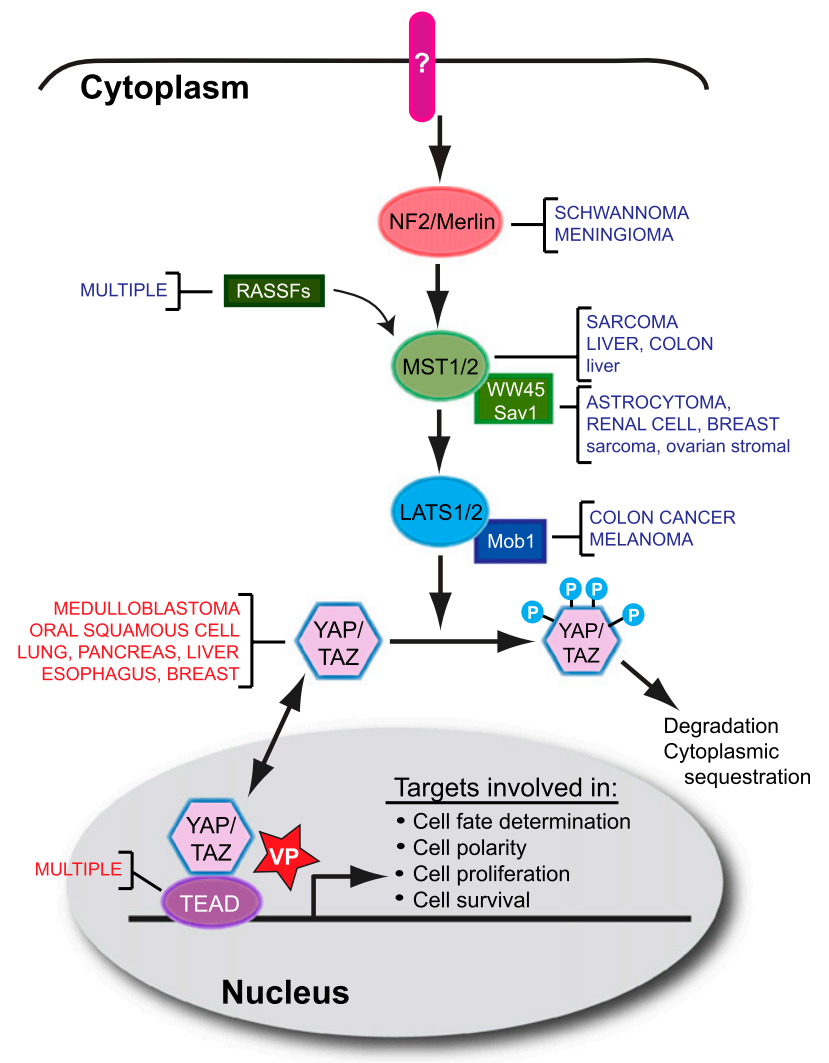

Figure 1. The mammalian Hippo pathway and cancer. A simplified view of the mammalian Hippo pathway reveals two sets of core kinases-Mst1/2 and Lats1/2-whose activity is modulated by the NF2/Merlin tumor suppressor, members of the Ras association domain family (RASSF), WW45/Sav1, and Mob1. When the pathway is "active," phosphorylation of the YAP or TAZ transcriptional coactivators by Lats $1 / 2$ results in degradation and/or cytoplasmic sequestration, while pathway "inactivation" allows unphosphorylated YAP/TAZ to enter the nucleus and bind one of four TEAD family members, resulting in context-dependent transcriptional output. In a variety of human (uppercase) and mouse (lowercase) cancers, upstream components of the pathway are down-regulated through frank deletion (NF2) or epigenetic mechanisms (decreased expression shown in blue). YAP, TAZ, and TEAD are up-regulated in a variety of human tumors (increased expression shown in red) by mechanisms that include gene amplification and silencing of upstream components of the pathway. The porphyrin molecule verteporfin (VP) disrupts the formation of the YAP-TEAD complex by binding to YAP and changing its conformation, thereby blocking the transcription of downstream targets.

vitro and in vivo (Overholtzer et al. 2006; Camargo et al. 2007; Dong et al. 2007) and is frequently amplified in a variety of human tumors (Baldwin et al. 2005; Snijders et al. 2005; Modena et al. 2006; Fernandez et al. 2009). In addition, YAP amplification has been found in breast cancer and hepatocellular carcinomas, where it is part of a chromosome 11q22 amplicon (Overholtzer et al. 2006; Zender et al. 2006). Similarly, high levels of TAZ expression have been reported in breast cancers /Chan et al. 2008), and both TEAD1 and TEAD4 have been reported 
to be amplified and/or up-regulated in a variety of cancers (for review, see Chan et al. 2010). Hence, a variety of mechanisms-gene amplification as well as down-regulation of upstream negative regulators-contribute to high levels of YAP, TAZ, and TEAD transcription factors in a wide spectrum of human tumors.

\section{Targeting the pathway}

The widespread involvement of the Hippo pathway in human cancer suggested early on that this pathway might be an attractive target for therapy. However, the fact that most parts of the pathway serve as negative regulators of the pathway's transcriptional output created a conceptual problem, since inhibitors of these components should result in YAP activation and hence promote cell proliferation. Against this backdrop, Liu-Chittenden et al. (2012) set out to address a number of key questions related to the tumorigenic activity of the Hippo pathway, which they now report in this issue of Genes \& Development. First, does the YAP/TAZ-TEAD transcription factor complex serve as the major (rate-limiting) conduit for the growthpromoting signals caused by loss of upstream components of the pathway, such as NF2/Merlin? Second, would it be possible to identify small molecules that can disrupt the formation of the TEAD-YAP complex in the nucleus? And finally, what are the consequences to normal tissue function of disrupting such a widely used pathway?

The investigators began by creating a dominant-negative version of TEAD2 in which the DNA-binding domain has been deleted, but YAP-TAZ binding is preserved (TEADDN). TEAD-DN was able to block YAP-mediated transcription in vitro as well as YAP's ability to promote anchorage-independent growth in soft agar. Then, to test the activity of their dominant-negative inhibitor in vivo, Liu-Chittenden et al. (2012) used an existing mouse model in which YAP overexpression could be induced in the liver in a doxycycline (Dox)-regulatable fashion; such YAPoverexpressing animals develop hepatomegaly after several weeks of Dox treatment and develop hepatocellular carcinoma (HCC) after 2 mo of Dox (Dong et al. 2007). In compound YAP/TEAD-DN mice, the investigators found that both the hepatomegaly phenotype and HCC formation were completely abrogated, indicating that the effects of YAP overexpression could be overcome by introducing an inhibitor that blocks the YAP-TEAD complex from binding DNA. To confirm that TEAD-DN could also inhibit YAP overexpression resulting from inactivation of an upstream component of the Hippo pathway, LiuChittenden et al. (2012) bred the genetic inhibitor to mice with a liver-specific deletion of NF2/Merlin, which also develop liver tumors (Zhang et al. 2010). Again, TEADDN prevented liver overgrowth and tumor formation.

Having concluded that disruption of the YAP-TEAD complex with a competitor protein could impede YAP's transforming activity, Liu-Chittenden et al. (2012) used a human embryonic kidney (HEK293) luciferase-based assay to screen a small molecule library for compounds that could inhibit YAP's transcriptional activity. Out of 71 hits that were able to inhibit YAP-mediated transcrip- tion at micromolar concentrations, they found three compounds (protoporphyrin IX, hematoporphyrin, and verteporfin [VP]) that had also been identified in a Drosophila interaction screen and were all members of the porphyrin family. As VP is already in clinical use (it is a photosensitizer used in photodynamic therapy for macular degeneration) (Michels and Schmidt-Erfurth 2001), the investigators focused their attention on this molecule, showing that VP inhibits the interaction between YAP and TEAD in coimmunoprecipitation experiments and that VP binds to purified YAP protein (but not TEAD2) in vitro. Proteolytic profiling experiments suggested that binding of VP to YAP changes YAP's conformation, rendering it incapable of binding to TEAD. Finally, the investigators used the two paradigms of liver overgrowth described earlierYAP overexpression and NF2/Merlin deletion-to test the efficacy of VP in vivo; in both cases, administration of $100 \mathrm{mg} / \mathrm{kg}$ VP to mice over a period of $8 \mathrm{~d}$ blocked the effects of pathway activation (overgrowth in the case of YAP overexpression, and biliary expansion in the case of NF2/Merlin deletion).

\section{Limitations and future considerations}

An important question for any tumor therapy, particularly one that targets a pathway as broadly active as the Hippo pathway, is the effect on normal tissues. In their study, Liu-Chittenden et al. (2012) provide some reassurance that inhibiting the pathway may be well tolerated, as overexpression of TEAD-DN in the liver had no effect on liver size, histology, or the expression of YAP target genes under normal (nononcogenic conditions). Moreover, a prior study by the same group, in which YAP was deleted in the intestine (using a conditional gene targeting approach), resulted in no overt effects on intestinal homeostasis (Cai et al. 2010), in contrast to the dramatic effects following YAP overexpression in the intestine (Camargo et al. 2007). However, the aforementioned measures of tissue "normalcy" remain fairly superficial and confined to a limited number of tissues; hence, a more extensive and robust investigation of the physiological roles played by the Hippo pathway has yet to be undertaken. Because the transcriptionally active mediators of the Hippo pathway consist of four DNA-binding proteins (TEAD1-4) and two coactivators (YAP and TAZ), it is possible that additional precision can be conferred by strategies that target specific combinations of interactions.

Another open question is whether disrupting the YAP/ TAZ-TEAD complex will be efficacious in the treatment of an established tumor. The studies performed by LiuChittenden et al. (2012) represent preventative trials in which the anti-tumor effects of the TEAD-DN or VP are exerted during early stages of tumor progression. It will be important to determine whether this compound and the more refined derivatives that are certain to follow have anti-tumor activity in the setting of an established tumor.

Although it is still in its early days, the work of LiuChittenden et al. (2012) is an important advance not only because it suggests a means of targeting the Hippo pathway, potentially for use in cancer therapy, but also 
because it is one of a limited number of studies indicating the viability of pharmacological approaches that target transcriptionally active complexes. For example, small molecules have been described that inhibit the formation of a complex between the Menin adaptor protein and fusion proteins containing the MLL histone methyltransferase, reversing the oncogenic activity of that complex (Grembecka et al. 2012). Similarly, small molecules that inhibit the ability of the bromodomain-containing protein Brd4 to bind to acetylated histones have broad activity against a number of leukemia cell lines (Zuber et al. 2011). Hence, we may be seeing the beginnings of a whole new arsenal of anti-tumor drugs: those that interfere with the formation of complexes that regulate the transcriptional output of a growth regulatory pathway, instead of solely inhibiting upstream enzymatic activities or ligand-receptor interactions.

\section{Conclusion}

When it comes to treating cancer, specificity is the name of the game. The newly identified Hippo pathway is activated in a wide variety of tumors, where it acts to promote cell proliferation and survival through the transcriptional activity of YAP-TEAD. Because the pathway is composed largely of negative regulators-kinases that inhibit the activity of YAP-it has been difficult to target, but Liu-Chittenden et al. (2012) have succeeded in developing genetic and pharmacological inhibitors by targeting the pathway's most downstream components: the YAP-TEAD complex itself. It remains to be seen whether pathway inhibition has efficacy in the treatment of established tumors and whether the agents that inhibit this pathway spare the function of normal tissues-the "holy grail" of targeted therapy.

\section{Acknowledgments}

I am grateful to Robert Vonderheide for helpful discussions.

\section{References}

Asthagiri AR, Parry DM, Butman JA, Kim HJ, Tsilou ET, Zhuang Z, Lonser RR. 2009. Neurofibromatosis type 2. Lancet 373: 1974-1986.

Baldwin C, Garnis C, Zhang L, Rosin MP, Lam WL. 2005. Multiple microalterations detected at high frequency in oral cancer. Cancer Res 65: 7561-7567.

Cai J, Zhang N, Zheng Y, de Wilde RF, Maitra A, Pan D. 2010. The Hippo signaling pathway restricts the oncogenic potential of an intestinal regeneration program. Genes Dev 24: 2383-2388.

Camargo FD, Gokhale S, Johnnidis JB, Fu D, Bell GW, Jaenisch R, Brummelkamp TR. 2007. YAP1 increases organ size and expands undifferentiated progenitor cells. Curr Biol 17: 2054-2060.

Chan SW, Lim CJ, Guo K, Ng CP, Lee I, Hunziker W, Zeng Q, Hong W. 2008. A role for TAZ in migration, invasion, and tumorigenesis of breast cancer cells. Cancer Res 68: 2592-2598.

Chan SW, Lim CJ, Chen L, Chong YF, Huang C, Song H, Hong W. 2010. The Hippo pathway in biological control and cancer development. J Cell Physiol 226: 928-939.
Dong J, Feldmann G, Huang J, Wu S, Zhang N, Comerford SA, Gayyed MF, Anders RA, Maitra A, Pan D. 2007. Elucidation of a universal size-control mechanism in Drosophila and mammals. Cell 130: 1120-1133.

Drebin JA, Link VC, Weinberg RA, Greene MI. 1986. Inhibition of tumor growth by a monoclonal antibody reactive with an oncogene-encoded tumor antigen. Proc Natl Acad Sci 83: 9129-9133.

Fernandez LA, Northcott PA, Dalton J, Fraga C, Ellison D, Angers S, Taylor MD, Kenney AM. 2009. YAP1 is amplified and up-regulated in hedgehog-associated medulloblastomas and mediates Sonic hedgehog-driven neural precursor proliferation. Genes Dev 23: 2729-2741.

Grembecka J, He S, Shi A, Purohit T, Muntean AG, Sorenson RJ, Showalter HD, Murai MJ, Belcher AM, Hartley T, et al. 2012. Menin-MLL inhibitors reverse oncogenic activity of MLL fusion proteins in leukemia. Nat Chem Biol 8: 277-284.

Halder G, Johnson RL. 2011. Hippo signaling: Growth control and beyond. Development 138: 9-22.

Justice RW, Zilian O, Woods DF, Noll M, Bryant PJ. 1995. The Drosophila tumor suppressor gene warts encodes a homolog of human myotonic dystrophy kinase and is required for the control of cell shape and proliferation. Genes Dev 9: 534546.

Lei QY, Zhang H, Zhao B, Zha ZY, Bai F, Pei XH, Zhao S, Xiong Y, Guan KL. 2008. TAZ promotes cell proliferation and epithelial-mesenchymal transition and is inhibited by the hippo pathway. Mol Cell Biol 28: 2426-2436.

Liu-Chittenden Y, Huang B, Shim JS, Chen Q, Lee SJ, Anders RA, Liu JO, Pan D. 2012. Genetic and pharmacological disruption of the TEAD-YAP complex suppresses the oncogenic activity of YAP. Genes Dev (this issue). doi: 10.1101/ gad.192856.112.

Michels S, Schmidt-Erfurth U. 2001. Photodynamic therapy with verteporfin: A new treatment in ophthalmology. Semin Ophthalmol 16: 201-206.

Modena P, Lualdi E, Facchinetti F, Veltman J, Reid JF, Minardi S, Janssen I, Giangaspero F, Forni M, Finocchiaro G, et al. 2006. Identification of tumor-specific molecular signatures in intracranial ependymoma and association with clinical characteristics. J Clin Oncol 24: 5223-5233.

Overholtzer M, Zhang J, Smolen GA, Muir B, Li W, Sgroi DC, Deng CX, Brugge JS, Haber DA. 2006. Transforming properties of YAP, a candidate oncogene on the chromosome 11q22 amplicon. Proc Nat1 Acad Sci 103: 12405-12410.

Pan D. 2010. The hippo signaling pathway in development and cancer. Dev Cell 19: 491-505.

Sawada A, Kiyonari H, Ukita K, Nishioka N, Imuta Y, Sasaki H. 2008. Redundant roles of Tead1 and Tead2 in notochord development and the regulation of cell proliferation and survival. Mol Cell Biol 28: 3177-3189.

Snijders AM, Schmidt BL, Fridlyand J, Dekker N, Pinkel D, Jordan RC, Albertson DG. 2005. Rare amplicons implicate frequent deregulation of cell fate specification pathways in oral squamous cell carcinoma. Oncogene 24: 4232-4242.

Xu T, Wang W, Zhang S, Stewart RA, Yu W. 1995. Identifying tumor suppressors in genetic mosaics: The Drosophila lats gene encodes a putative protein kinase. Development 121: 1053-1063.

Zender L, Spector MS, Xue W, Flemming P, Cordon-Cardo C, Silke J, Fan ST, Luk JM, Wigler M, Hannon GJ, et al. 2006. Identification and validation of oncogenes in liver cancer using an integrative oncogenomic approach. Cell 125: 12531267.

Zhang N, Bai H, David KK, Dong J, Zheng Y, Cai J, Giovannini M, Liu P, Anders RA, Pan D. 2010. The Merlin/NF2 tumor 
suppressor functions through the YAP oncoprotein to regulate tissue homeostasis in mammals. Dev Cell 19: 27-38.

Zhao B, Wei X, Li W, Udan RS, Yang Q, Kim J, Xie J, Ikenoue T, $\mathrm{Yu}$ J, Li L, et al. 2007. Inactivation of YAP oncoprotein by the Hippo pathway is involved in cell contact inhibition and tissue growth control. Genes Dev 21: 2747-2761.

Zhao B, Li L, Lei Q, Guan KL. 2010a. The Hippo-YAP pathway in organ size control and tumorigenesis: An updated version. Genes Dev 24: 862-874.

Zhao B, Li L, Tumaneng K, Wang CY, Guan KL. 2010b. A coordinated phosphorylation by Lats and CK1 regulates YAP stability through SCF( $\beta$-TRCP). Genes Dev 24: 72-85.

Zuber J, Shi J, Wang E, Rappaport AR, Herrmann H, Sison EA, Magoon D, Qi J, Blatt K, Wunderlich M, et al. 2011. RNAi screen identifies Brd4 as a therapeutic target in acute myeloid leukaemia. Nature 478: 524-528. 


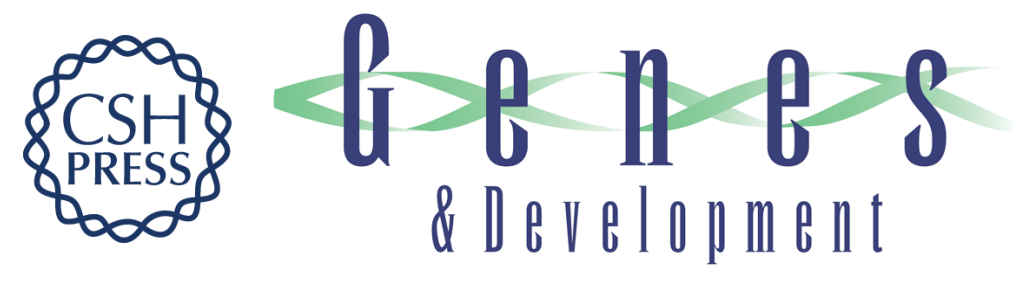

\section{Quit your YAPing: a new target for cancer therapy}

\section{Ben Z. Stanger}

Genes Dev. 2012, 26:

Access the most recent version at doi:10.1101/gad.196501.112

\section{Related Content Genetic and pharmacological disruption of the TEADYAP complex suppresses the oncogenic activity of YAP \\ Yi Liu-Chittenden, Bo Huang, Joong Sup Shim, et al. \\ Genes Dev. June, 2012 26: 1300-1305}

$\begin{array}{ll}\text { References } & \text { This article cites } 26 \text { articles, } 15 \text { of which can be accessed free at: } \\ \text { http://genesdev.cshlp.org/content/26/12/1263.full.html\#ref-list-1 }\end{array}$

Articles cited in:

http://genesdev.cshlp.org/content/26/12/1263.full.html\#related-urls

\section{License}

Email Alerting Receive free email alerts when new articles cite this article - sign up in the box at the top Service right corner of the article or click here.

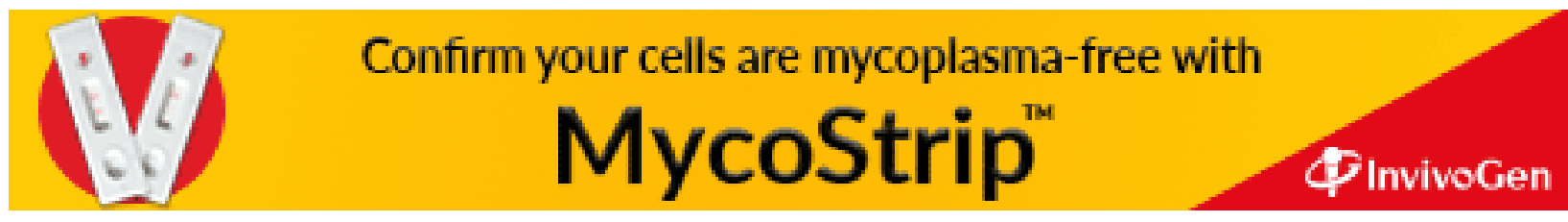

